\title{
Authors' Index
}

\section{ABE, K. 195}

ANAND KUMAR, T.C. 114

ARIMURA, A. 121

BATtA, S.K. 224 Bohus, B. 280 BRandstateR, J.F. 362 BRIEL, G. 34 BRown, G.M. 351

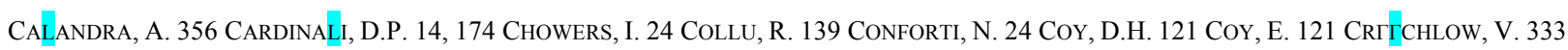

Dames, W. 34 David, G.F.X. 114 Davidson, J.M. 129, 369 DeBeLJuK, L. 121 DöcKE, F. 345 DominguEZ, R. 212 Ducharme, J.R. 139

FAHRINGeR, E.E. 151 FeldMan, S. 24 FLeRKó, B. 187 Foley, E.L. 151

Garcia BiÉnere, W. 174 GibBS, CARol 72 Gona, A.G. 365 Gona, O. 365 Gorski R.A. 321 GreEnStein, M. 95, 165

HAYASHI, S. 321

HEWITT, ROSEMARY 233 HIROSHIGE, T. 195

ILLEI-DONHOFFER, AGNES 187

JACOBY, J.H. 95,165 JÉQ́UIER, J.-C. 139 JOHNSON, J.H. 103 JOHNSON, LINDA Y. 310 JONG, W. DE 297

KASTIN, A.J. 271

LABorde, N.P. 174 Lazarus, L. 362 Leboeuf, G. 139 Letarte, J. 139

Mann, Brigitte G. 65 Maricchiolo, M. 356 Martini, L. 224 Maurer, R.A. 87 Mennin, S.P. 321 Mess, B. 187 Miller, R.E. 233, 257 Minard, F.N, 271

NAGLE, C.A. 14, 174 NeQUiN, L.G. 65 NeUHofF, V. 34 NIR, I. 34

OLSON, J. 1

Palkovits, M. 297 Pearlmutter, A. Frances 257 Pedroza Garcia, E. 174 Pezzino, V. 356 Plotnikoff, N.P. 271 Purves, H.D. 251

RAmaley, Judith A. 1 Redgate, E.S. 151 Reinhardt, V. 289 ReIr

Sassin. J.F. 95, 165 Sawyer, C.H. 103 SChally, A.V. 121 Schams, D. 289 Schwartz, NeEna B. 65 Seggie, J. 351 Siegel, R. 24 Sirett, Nancy E. 251 Smith, ERLa R. 129 , 212 , 369 Smythe, G.A. 362 Squatrito, S. 356 StePhan, F.K. 44

Authors' Index

UhLIIR, I. 351

VIGNERI, R. 356 VILCHEZ-MARTINEZ, J.A. 121

Welsz, Judith 72 Weitzman, E.D. 95, 165 WhгTMOYer, D.I. 103 WIIED, D. DE 280, 297 Wiley, M. KATHLEen 233, 257 WiLSON, MarLene 333 WiMERSMA GRETDANUS, Tj.B. VAN 280

WOOLLEY, DOROTHY E. 87

YANAIHARA, N. 121 YUEh-ChIIEN, H. 233

ZANISI, M. 224 ZUCKER, I. 44

TALLEY, W.L. 65 TerKEL, J. 103 